\title{
Artificial Inteilligence Trends That Will Dominate 2018
}

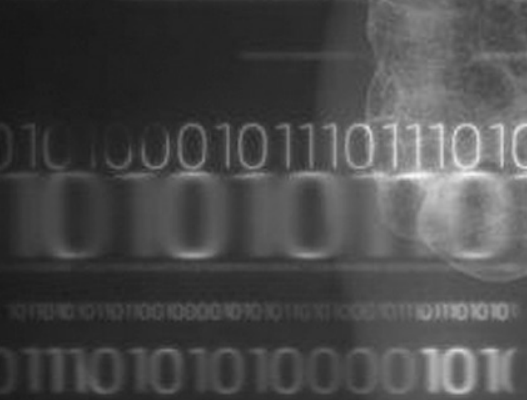

\section{Everyone is talking about machine learning, deep learning,}

\section{and Al. Here are five Al trends to watch in 2018}

2017 saw an explosion of machine learning in production use, with even deep learning and artificial intelligence (Al) being leveraged for practical applications.

«Basic analytics are out; machine learning (and beyond) are in.

The practical applications of machine learning, deep learning, and $\mathrm{Al}$ are "everywhere and out in the open these days (including the make and model of passing cars) to deliver targeted advertisements.

Cutting through the hype with our practical guide to machine learning in business and find out whether your organization is truly ready for taking on artificial intelligence projects.

So where will these frameworks and tools take us in 2018 ? As already foreseen in the recent issued

\section{* Enterprises will operationalize Al}

$\mathrm{Al}$ is already here, whether we recognize it or not. Many organizations are already using Al, but they may not refer to it as «Al, «For example, any organization using a chatbot feature to engage with customers is using artificial intelligence.»

But many of the deployments leveraging Al technologies and tools have been small-scale. Expect organizations to ramp up in a big way in 2018. Enterprises have spent the past few years educating themselves on various Al frameworks and tools «But as Al goes mainstream, it will move beyond small-scale experiments to being automated and operationalized. As enterprises move forward with operationalizing Al, they will look for products and tools to automate, manage, and streamline the entire machine learning and deep learning life cycle.» 
It is predicted that 2018 will see an increase in investments in Al life cycle management, and technologies that house the data and supervise the process will mature.

\section{* Al reality will lag the hype once again}

There have been repeated predictions for several years that tout potential breakthroughs in the use of Al and machine learning, but the reality is that most enterprises have yet to see quantifiable benefits from their investments in these areas.

Chen says the hype to date has been overblown, and most enterprises are reluctant to get started due to a combination of skepticism, lack of expertise, and most important of all, a lack of confidence in the reliability of their data sets.

In fact, while the headlines will be mostly about Al, most enterprises will need to first focus on IA (information augmentation): getting their data organized in a manner that ensures it can be reconciled, refined, and related, to uncover relevant insights that support efficient business execution across all departments, while addressing the burden of regulatory compliance..

It is agreed in many research works that 2018 will see a backlash against $\mathrm{Al}$ hype, but believes a more balanced approach of deep learning and shallow learning application to business opportunities will emerge as a result.

While there may be a backlash against the hype, it would not stop large enterprises from investing in $\mathrm{Al}$ and related technologies.

Meley points to Teradata's recently released 2017 State of Artificial Intelligence for Enterprises report, which identified a lack of IT infrastructure as the greatest barrier to realizing benefits from $\mathrm{Al}$, surpassing issues like access to talent, lack of budget, and weak or unknown business cases.

«Companies will respond in 2018 with enterprise-grade Al product and supporting offerings that overcome the growing pains associated with $\mathrm{Al}$ adoption,» Meley says.

\section{* Bias in training data sets will continue to trouble $\mathrm{Al}$}

Enterprises need to get their data in order, which is a driving force behind the open source Apache Arrow project, believes a debate about data sets will take center stage in 2018.

Everywhere you turn, companies are adding $\mathrm{Al}$ to their products to make them smarter, more efficient, and even autonomous. In 2017, competing arguments for whether Al would create jobs or eliminate them, with some even proposing the end of the human race. What has started to emerge as a key part of the conversation is how training data sets shape the behavior of these models.

It turns out, that models are only as good as the training data they use, and developing a representative, effective training data set is very challenging.

Humans are hopelessly biased, and the question for $\mathrm{Al}$ will become whether we can do better in terms of bias or will we do worse. This debate will center around data ownership - what data we own about ourselves, and the companies like Google, Facebook, Amazon, Uber, etc. who have amassed enormous data sets that will feed our models.

\section{* Al must solve the 'black box' problem with audit trails}

One of the big barriers to the adoption of $\mathrm{Al}$, particularly in regulated industries, is the difficulty in showing exactly how an $\mathrm{Al}$ reached a decision, therefore, creating $\mathrm{Al}$ audit trails will be essential.

$\mathrm{Al}$ is increasingly getting used for applications like drug discovery or the connected car, and these applications can have a detrimental impact on human life if an incorrect decision is made. Detecting exactly what caused the final incorrect decision leading to a serious problem is something enterprises will start to look at in 2018. Auditing and tracking every input and every score that a framework produces will help with detecting the human-written code that ultimately caused the problem.

\section{* Cloud adoption will accelerate to sup- port Al innovation}

Enterprises in 2018 will seek to improve their infrastructure and processes for supporting their machine learning and $\mathrm{Al}$ efforts.

As companies look to innovate and improve with machine learning and artificial intelligence, more specialized tooling and infrastructure will be adopted in the cloud to support specific use cases, like solutions for merging multi-modal sensory inputs for human interaction (think sound, touch, and vision) or solutions for merging satellite imagery with financial data to catapult algorithmic trading capabilities.

It is expected to see an explosion in cloud-based solu- 
tions that accelerate the current pace of data collection and further demonstrate the need for frictionless, on-demand compute and storage from managed cloud providers.

2017 saw an explosion of machine learning in production use, with even deep learning and artificial intelligence (Al) is leveraged for practical applications.

«Basic analytics are out; machine learning (and beyond) are in.

Practical applications of machine learning, deep learning, and $\mathrm{Al}$ are «everywhere and out in the open these days, that leverage hidden cameras gathering data on foot and road traffic (including the make and model of passing cars) to deliver targeted advertisements.

Cutting through the hype with our practical guide to machine learning in business and find out whether your organization is truly ready for taking on artificial intelligence projects.

So where will these frameworks and tools take us in 2018 ? We spoke with a number of IT leaders and industry experts about what to expect in the coming year.

\section{* Top Trends of Mind When Crafting an Al Strategy}

According to a survey of 83 Gartner clients, $60 \%$ of respondents reported to be in an $\mathrm{Al}$ «knowledge-gathering phase," $25 \%$ said they are piloting an $\mathrm{Al}$ solution and a mere $5 \%$ of respondents said they have implemented an Al solution.

\section{* Natural and contextual interfaces}

New interfaces will dramatically change the way consumers and employees access computing resources, Andrews said. Specifically, the new wave of interfaces relies on natural language processing and generation, visual analytics and gesture interpretation -- technologies powered by Al. They will play an increasingly important role in enterprise interfaces.

In a client example Andrews titled the «Warehouse of Babel,» artificial intelligence is bridging a language barrier for a European-based warehouse. The warehouse is now using a natural language interpretation system so that employees, who come from all corners of Eastern Europe, donst have to speak the same language to communicate or access applications «in a comparatively unified way,» Andrews said.

These natural and contextual interfaces are being em- bedded into consumer products right now -- Amazon Echo and Google Glass are two examples. As these offerings proliferate, they will raise employee expectations for workplace tech, Andrews said, with good reason. «[These new interfaces provide] a more effective way of allowing people to use $\mathrm{Al}$ as a means of interacting with content, with social situations and systems, with business applications, with data and with documents, " he said.

\section{* Smarter IoT and better application in- tegration}

Al capabilities are being embedded into the internet of things (IoT) devices that operate on the computing edge, but those capabilities will be limited. Model building with $\mathrm{Al}$ will happen elsewhere, but runtime analysis and «interaction into action models» that provide, say, visual analysis can live on an edge device, Andrews said.

Al technologies affect all corners of business, IT

For example, Raspberry $\mathrm{Pi}$, an inexpensive microcomputer, can use basic visual analytics to recognize colors and shapes. And in the Warehouse of Babel example, Andrews said the client is looking to invest in a visual analytics application to more efficiently load shipping containers and reduce unused space. «I think of this as being like Tetris,» he said.

Indeed, Gartner predicts that by 2022 , more than $80 \%$ of enterprise IoT projects will have an Al component. That's up from less than $10 \%$ today.

Companies are also beginning to integrate $\mathrm{Al}$ into existing applications to create a more fluid experience, predominately for customer-engagement applications and call center service and support applications, according to a Gartner survey.

The net effect? It's the beginning of «an Al ecosystem that is intriguingly complex, " Andrews said. He expects the pattern to continue, especially for early adopters who, according to a Gartner prediction, will use four virtual personal assistants on average by 2022 .

\section{* Rise of Al-enabled computing ecosys- tems}

Al-powered applications will be able to tell each other what they need to meet a goal without human interaction. But to create this kind of commonplace Al, application diversity is crucial. «In any ecosystem, strength comes from that diversity and from multiple perspectives,» he said. «lt,s something extraordinary that allows a system to be resilient.» 